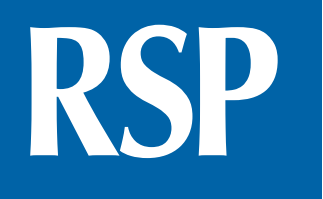

http://www.rsp.fsp.usp.br/
Revista de Saúde Pública

\title{
Fatores associados à prematuridade em casos notificados de sífilis congênita
}

\author{
Maria Alix Leite Araújo' (iD, Ana Beatriz Barbosa Esteves" iD, Ana Fátima Braga Rocha"I' (iD, \\ Geraldo Bezerra da Silva Junior ${ }^{\mathrm{Iv}}$ iD, Angelica Espinosa Miranda ${ }^{\mathrm{v}}$ (D) \\ I Universidade de Fortaleza. Programa de Pós-Graduação em Saúde Coletiva. Fortaleza, CE, Brasil \\ " Universidade de Fortaleza. Programa de Pós-Graduação em Saúde Coletiva. Fortaleza, CE, Brasil \\ III Faculdade Terra Nordeste. Curso de Bacharelado em Enfermagem. Caucaia, CE, Brasil \\ iv Universidade de Fortaleza. Programa de Pós-Graduação em Saúde Coletiva. Fortaleza, CE, Brasil \\ $\checkmark$ Universidade Federal do Espírito Santo. Departamento de Medicina Social. Vitoria, ES, Brasil
}

\author{
Correspondência: \\ Maria Alix Leite Araújo \\ Av. Washington Soares, 1321 - Edson \\ Queiroz \\ 60811-905 Fortaleza, CE, Brasil \\ E-mail: mleite@unifor.br
}

Recebido: 21 fev 2020

Aprovado: 2 set 2020

Como citar: Araujo MAL, Esteves ABB, Rocha AFB, Silva Junior GB, Miranda AE. Fatores associados à prematuridade em casos notificados de sífilis congênita. Rev Saude Publica. 2021;55:28. https://doi.org/10.11606/s15188787.2021055002400

Copyright: Este é um artigo de acesso aberto distribuído sob os termos da Licença de Atribuição Creative Commons, que permite uso irrestrito, distribuição e reprodução em qualquer meio, desde que o autor e a fonte originais sejam creditados.

\section{RESUMO}

OBJETIVO: Analisar os fatores associados à prematuridade em casos notificados de sífilis congênita no município de Fortaleza, Ceará, Brasil.

MÉTODOS: Estudo transversal realizado em dez maternidades públicas de Fortaleza, Ceará, Brasil. Foram incluídos 478 casos notificados de sífilis congênita em 2015, e os dados foram coletados das fichas de notificação, dos prontuários das mães e dos bebês e do cartão de pré-natal. Para a análise bivariada, foram utilizados os testes do qui-quadrado de Pearson e exato de Fisher, considerando $\mathrm{p}<0,05$. Realizou-se regressão logística múltipla, apresentando razão de chances (OR) com intervalo de confiança de $95 \%$.

RESULTADOS: Encontrou-se 15,3\% de prematuridade em gestantes com sífilis. A titulação do teste VDRL > 1:8 no parto (OR 2,46; IC95\%: 1,33-4,53; p = 0,004), o não tratamento da gestante ou tratamento realizado com drogas diferentes da penicilina durante o pré-natal (OR 3,52; IC95\%: 1,74-7,13; $\mathrm{p}<0,001)$ estiveram associados a maiores chances de prematuridade.

CONCLUSÃO: A prematuridade decorrente da sífilis congênita é um agravo evitável, desde que as gestantes com sífilis sejam tratadas adequadamente. As fragilidades na assistência pré-natal estão associadas a este desfecho, o que ressalta a importância de implementar políticas públicas voltadas a melhorar a qualidade do pré-natal.

DESCRITORES: Sífilis Congênita. Transmissão Vertical de Doença Infecciosa. Cuidado Pré-Natal. Recém-Nascido Prematuro. Penicilina G. 


\section{INTRODUÇÃO}

A sífilis é uma infecção sistêmica que, quando acomete a gestante, pode ser transmitida ao bebê, causando a sífilis congênita (SC), com consequências graves, dentre elas a prematuridade $^{1}$. A etiologia do parto prematuro é multifatorial, e as causas infecciosas, dentre elas as infecções sexualmente transmissíveis (IST), são relevantes, tendo em vista sua alta prevalência e associação, quando não tratadas, a desfechos obstétricos indesejáveis² Neste contexto, a sífilis assume protagonismo ${ }^{3}$.

Segundo a Organização Mundial da Saúde (OMS), em 2016, cerca de 1 milhão de gestantes estavam infectadas com sífilis, o que teve como consequência crianças com manifestações clínicas precoces e tardias da SC ${ }^{4}$. Nas Américas, a taxa de incidência de $\mathrm{SC}$ vem crescendo ao longo dos anos, e os casos notificados no Brasil contribuíram consideravelmente para esse aumento ${ }^{5}$.

No Brasil, somente em 2018, foram notificados 158.051 casos de sífilis adquirida, 62.599 casos de sífilis em gestantes, e 26.219 de SC. O estado do Ceará está dentre aqueles que apresentaram as maiores taxas do país 6 , e sua capital, Fortaleza, notificou nesse mesmo ano 827 casos de $\mathrm{SC}^{7}$, com taxa de incidência sempre crescente ao longo dos anos ${ }^{8}$.

Considerando a gravidade dos desfechos, medidas de controle têm sido recomendadas 9 . A redução da SC está contemplada nos Objetivos para o Desenvolvimento Sustentável, que buscam eliminar as mortes evitáveis de recém-nascidos e crianças menores de 5 anos, reduzir a mortalidade neonatal e combater as infecções transmissíveis ${ }^{10}$. Neste contexto, a prematuridade tem destaque, considerando que ela é a principal causa de morte em crianças menores de 5 anos no mundo ${ }^{11,12}$, contribuindo para aumentar a mortalidade neonatal em países pobres e em desenvolvimento ${ }^{13}$.

Diante dos aspectos supracitados, e considerando que ainda persistem lacunas importantes nos estudos acerca da prematuridade por SC, este artigo tem por objetivo analisar os fatores associados à prematuridade em casos notificados de SC no município de Fortaleza, Ceará, Brasil.

\section{MÉTODOS}

Este estudo transversal, que analisou a prevalência e os fatores associados ao desfecho de prematuridade em gestantes com sífilis, utilizou dados de todas as dez maternidades públicas do município de Fortaleza, capital do estado do Ceará.

Fortaleza tem população de cerca de 2,5 milhões de habitantes, produto interno bruto de $\mathrm{R} \$ 41,2$ bilhões, e índice de desenvolvimento humano de $0,754^{14}$. A cobertura da Estratégia Saúde da Família é de 57,6\%, e a de pré-natal de 95\% (dados não publicados, disponibilizados pela Secretaria Municipal de Saúde).

Fizeram parte do estudo todos os casos notificados de SC em 2015, considerando crianças que nasceram vivas, isto é, partos em que houve expulsão ou extração completa do feto, independentemente da idade gestacional da mãe, do produto da concepção e da apresentação, depois da separação materna, de qualquer sinal de vida (batimentos cardíacos, respiração ou até mesmo pulsação do cordão umbilical). Para definir a SC, foram considerados os seguintes critérios, estabelecidos pelo Ministério da Saúde ${ }^{15}$ (p. 26):

- Criança cuja mãe apresente, durante o pré-natal ou no momento do parto, testes para sífilis não treponêmico reagente com qualquer titulação e treponêmico reagente, e que não tenha sido tratada ou tenha recebido tratamento inadequado.

- Criança cuja mãe não foi diagnosticada com sífilis durante a gestação e, na impossibilidade de a maternidade realizar o teste treponêmico, apresente teste não treponêmico reagente com qualquer titulação no momento do parto. 
- Criança cuja mãe não foi diagnosticada com sífilis durante a gestação e, na impossibilidade de a maternidade realizar o teste não treponêmico, apresente teste treponêmico reagente no momento do parto.

- Criança cuja mãe apresente teste treponêmico reagente e teste não treponêmico não reagente no momento do parto, sem registro de tratamento prévio.

Foram excluídos os casos de abortos e natimortos, devido à indisponibilidade de informações para muitas das variáveis inseridas neste estudo, o que prejudicaria a análise. Além disso, também foram excluídos: casos de gravidez ectópica, mola hidatiforme ou aborto, e casos em que as gestantes apresentaram cardiopatias, pneumopatias graves, nefropatias graves, endocrinopatias, doenças hematológicas e hipertensão arterial crônica, doenças neurológicas, doenças psiquiátricas que necessitam de acompanhamento, doenças autoimunes, alterações genéticas, antecedente de trombose venosa profunda ou embolia pulmonar, doenças ginecológicas, hanseníase, tuberculose ou qualquer patologia clínica que necessite de acompanhamento especializado, bem como casos de pacientes que fazem uso de anti-hipertensivo ( $\mathrm{PA}>140 / 90 \mathrm{mmHg}$ antes de 20 semanas de idade gestacional - IG). A exclusão desses agravos ocorreu por estarem relacionadas a risco de prematuridade $e^{6-8}$, podendo atuar como fator de confundimento para prematuridade por SC.

A coleta de dados foi realizada entre os meses de janeiro e novembro de 2018. Foram levantados todos os casos de SC notificados em 2015 no Sistema de Informação de Agravos de Notificação (Sinan). Os dados foram colhidos das fichas de notificação e, posteriormente, dos prontuários médicos da mãe e do bebê e do cartão de pré-natal da gestante. Vale salientar que as maternidades anexam uma cópia do cartão da gestante ao prontuário. Quando havia divergências entre as fontes de dados, foram considerados válidos os dados dos prontuários.

O desfecho analisado foi prematuridade (quando a criança nasce com menos de 37 semanas completas de gestação). Analisaram-se as seguintes variáveis maternas: sociodemográficas (idade, situação conjugal, escolaridade, ocupação) e relacionadas à assistência pré-natal (frequentou o pré-natal, número de consultas, início do acompanhamento, realização dos exames para a sífilis, número de exames para sífilis, resultado do primeiro e segundo exames para sífilis, titulação do teste VDRL no pré-natal, momento do diagnóstico de sífilis, tratamento da gestante no pré-natal, tratamento do parceiro e titulação do teste VDRL no parto) e uso de drogas ilícitas.

Os dados foram analisados no programa estatístico SPSS (Statistical Package for the Social Sciences, versão 23, IBM, USA) e no Stata versão 10.0 (Stata Corp., USA), pela técnica de Stepwise para regressão logística múltipla. Para a análise bivariada, utilizou-se o teste qui-quadrado de Pearson e teste exato de Fisher, considerando $p<0,05$. A análise ajustada incluiu as variáveis com valor de $\mathrm{p}<0,20$, permanecendo as que tiveram valor de $\mathrm{p}<0,05$. Como medida de efeito, utilizou-se a razão de chances (OR) e o intervalo de confiança de $95 \%$.

Este estudo foi aprovado pelo Comitê de Ética e Pesquisa da Universidade de Fortaleza (Unifor), sob o Parecer no 2.110.189, e faz parte de uma pesquisa maior, intitulada "Manifestações Clínicas e Complicações em Crianças com Sífilis Congênita”.

\section{RESULTADOS}

No ano de 2015, foram notificados 674 casos de SC em Fortaleza, dos quais 478 foram considerados elegíveis. Excluíram-se 196 casos (31 abortos, 39 natimortos e 126 gestantes que apresentaram outros agravos de risco para prematuridade). Nasceram prematuras 73 crianças (15,3\%). 
Tabela 1. Perfil sociodemográfico e obstétrico de gestantes com sífilis com desfecho de prematuridade (Fortaleza, Ceará, 2015).

\begin{tabular}{|c|c|c|}
\hline Variáveis & $\mathbf{n}^{\mathrm{a}}$ & $\%$ \\
\hline \multicolumn{3}{|l|}{ Idade (em anos) } \\
\hline$\leq 19$ & 15 & 20,5 \\
\hline $20-29$ & 41 & 56,2 \\
\hline$\geq 30$ & 17 & 23,3 \\
\hline \multicolumn{3}{|l|}{ Situação conjugal } \\
\hline Com parceiro fixo & 36 & 49,3 \\
\hline Sem parceiro fixo & 37 & 50,7 \\
\hline \multicolumn{3}{|l|}{ Escolaridade (anos de estudo) } \\
\hline Até ensino fundamental completo & 54 & 74,0 \\
\hline Ensino médio incompleto/completo/superior & 19 & 26,0 \\
\hline \multicolumn{3}{|l|}{ Possui trabalho remunerado } \\
\hline Sim & 13 & 17,8 \\
\hline Não & 60 & 82,2 \\
\hline \multicolumn{3}{|l|}{ Usuária de drogas ilícitas } \\
\hline Sim & 26 & 35,6 \\
\hline Não/ignorado & 47 & 64,4 \\
\hline \multicolumn{3}{|l|}{ Frequentou pré-natal } \\
\hline Sim & 52 & 71,2 \\
\hline Não & 21 & 28,8 \\
\hline \multicolumn{3}{|l|}{ Número de consultas no pré-natal } \\
\hline 1 & 8 & 15,4 \\
\hline 2 a 5 & 26 & 50,0 \\
\hline$\geq 6$ & 18 & 34,6 \\
\hline \multicolumn{3}{|l|}{ Início do pré-natal } \\
\hline $1^{\circ}$ trimestre & 23 & 44,2 \\
\hline $2^{\circ}$ trimestre & 20 & 38,5 \\
\hline $3^{\circ}$ trimestre & 9 & 17,3 \\
\hline \multicolumn{3}{|l|}{ Número de exames para sífilis no pré-natal } \\
\hline Nenhum & 15 & 28,9 \\
\hline 1 & 31 & 59,6 \\
\hline 2 & 6 & 11,5 \\
\hline \multicolumn{3}{|l|}{ Resultado do exame para sífilis no pré-natalb } \\
\hline Reagente & 21 & 56,8 \\
\hline Não reagente & 16 & 43,2 \\
\hline \multicolumn{3}{|l|}{ Titulação do VDRL no pré-natal ${ }^{\mathrm{c}}$} \\
\hline$\leq 1: 8$ & 4 & 30,8 \\
\hline$>1: 8$ & 9 & 69,2 \\
\hline \multicolumn{3}{|l|}{ Tratamento realizado no pré-natal } \\
\hline Tratamento completo $^{d}$ & 4 & 7,7 \\
\hline Tratamento incompleto & 7 & 13,5 \\
\hline Outra droga/Não tratada & 41 & 78,8 \\
\hline \multicolumn{3}{|l|}{ Titulação do VDRL do parto } \\
\hline$\leq 1: 8$ & 41 & 56,2 \\
\hline$>1: 8$ & 32 & 43,8 \\
\hline
\end{tabular}

a Nem todas as pacientes realizaram pré-natal ou todos os exames para sífilis, por isso o "n" de algumas variáveis é diferente.

b Teste rápido ou exame de VDRL.

c Apenas para aquelas que realizaram exame de VDRL.

${ }^{\mathrm{d}}$ Tratamento com três doses de penicilina benzatina (7,2 milhões UI) independente do tratamento do parceiro. 
O perfil sociodemográfico e obstétrico das gestantes com sífilis que tiveram bebês prematuros está apresentado na Tabela 1 . A média de idade das gestantes foi de 24,9 anos (DP $=6,3$ ), e a maioria tinha entre 20 e 29 anos de idade; 54 (74\%) completaram o ensino fundamental e $26(35,6 \%)$ referiram uso de alguma droga ilícita. Cinquenta e duas mulheres frequentaram o pré-natal $(71,2 \%)$; destas, $37(71,1 \%)$ realizaram pelo menos um exame para sífilis; e $11(21,2 \%)$ realizaram tratamento com pelo menos uma dose de penicilina benzatina. Na ocasião do parto, $32(43,8 \%)$ apresentaram titulação > 1:8 no exame de VDRL (Tabela 1).

Os dados de gestantes cuja titulação do VDRL foi > 1:8 no momento do parto estão descritos na Tabela 2. Dentre as gestantes com sífilis que tiveram bebês prematuros, $24(75 \%)$ frequentaram o pré-natal; destas, $9(37,5 \%)$ não realizaram nenhum exame para sífilis. Todas que apresentaram resultado reagente no pré-natal tiveram titulação > 1:8. Os casos de prematuridade ocorreram em gestantes que realizaram tratamento incompleto e, principalmente, que não foram tratadas ou receberam outra droga diferente da penicilina.

Na análise bivariada, mostraram associação com o desfecho de prematuridade: uso de droga ilícita ( $\mathrm{p}=0,001$; OR: 2,39; IC95\%: 1,39-4,11), não ter frequentado o pré-natal ( $\mathrm{p}=0,004$; OR:

Tabela 2. Análise da assistência pré-natal de gestantes que apresentaram titulação de VDRL > 1:8 no momento do parto (Fortaleza, Ceará, 2015).

\begin{tabular}{|c|c|c|c|c|}
\hline \multirow{3}{*}{ Variáveis } & \multicolumn{4}{|c|}{ VDRL parto > 1:8 } \\
\hline & \multicolumn{2}{|c|}{$\begin{array}{c}\text { Total de casos sífilis congênita } \\
(n=134)\end{array}$} & \multicolumn{2}{|c|}{$\begin{array}{c}\text { Prematuridade } \\
(\mathrm{n}=32)\end{array}$} \\
\hline & $\mathbf{n}^{\mathrm{a}}$ & $\%$ & $\mathbf{n}^{\mathrm{a}}$ & $\%$ \\
\hline \multicolumn{5}{|l|}{ Frequentou pré-natal } \\
\hline Sim & 111 & 82,8 & 24 & 75,0 \\
\hline Não & 23 & 17,2 & 8 & 25,0 \\
\hline \multicolumn{5}{|c|}{ Número de consultas no pré-natal } \\
\hline 1 & 8 & 7,2 & 5 & 20,8 \\
\hline 2 a 5 & 53 & 47,7 & 13 & 54,2 \\
\hline$\geq 6$ & 50 & 45,0 & 6 & 25,0 \\
\hline \multicolumn{5}{|l|}{ Início do pré-natal } \\
\hline $1^{\circ}$ trimestre & 49 & 44,2 & 8 & 33,3 \\
\hline $2^{\circ}$ trimestre & 45 & 40,5 & 10 & 41,7 \\
\hline $3^{\circ}$ trimestre & 17 & 15,3 & 6 & 25,0 \\
\hline \multicolumn{5}{|c|}{ Número de exames para sífilis no pré-natal ${ }^{b}$} \\
\hline Nenhum & 28 & 25,2 & 9 & 37,5 \\
\hline 1 & 65 & 58,6 & 13 & 54,2 \\
\hline 2 & 18 & 16,2 & 2 & 8,3 \\
\hline \multicolumn{5}{|c|}{ Resultado do exame para sífilis no pré-natal ${ }^{b}$} \\
\hline Reagente & 65 & 78,3 & 6 & 40,0 \\
\hline Não reagente & 18 & 21,7 & 9 & 60,0 \\
\hline \multicolumn{5}{|c|}{ Titulação do VDRL no pré-natal ${ }^{\mathrm{c}}$} \\
\hline$\leq 1: 8$ & 13 & 23,6 & - & - \\
\hline$>1: 8$ & 42 & 76,4 & 6 & 100,0 \\
\hline \multicolumn{5}{|c|}{ Tratamento realizado no pré-natal } \\
\hline Tratamento complet ${ }^{\circ} \mathrm{d}$ & 24 & 21,6 & - & - \\
\hline Tratamento incompleto & 22 & 19,8 & 3 & 12,5 \\
\hline Outra droga/não tratada & 65 & 58,6 & 21 & 87,5 \\
\hline
\end{tabular}

a Nem todas as pacientes realizaram pré-natal ou todos os exames para sífilis, por isso o " $\mathrm{n}$ " de algumas variáveis é diferente.

' Teste rápido e/ou exame de VDRL.

${ }^{c}$ Apenas para aquelas que realizaram exame de VDRL.

${ }^{\mathrm{d}}$ Tratamento com três doses de penicilina benzatina (7,2 milhões UI), independente do tratamento do parceiro. 
Tabela 3. Aspectos sociodemográficos, do pré-natal e do parto de gestantes com sífilis associados ao desfecho de prematuridade (Fortaleza, Ceará, 2015).

\begin{tabular}{|c|c|c|c|c|c|c|c|}
\hline \multirow{3}{*}{ Variáveis } & \multirow{3}{*}{ n $(\%)$} & \multicolumn{4}{|c|}{ Prematuridade } & \multirow{3}{*}{$\mathbf{p}$} & \multirow{3}{*}{ OR bruto (IC95\% } \\
\hline & & \multicolumn{2}{|c|}{ Sim } & \multicolumn{2}{|c|}{ Não } & & \\
\hline & & $\mathbf{n}$ & $\%$ & $\mathbf{n}$ & $\%$ & & \\
\hline Idade (em anos) & & & & & & 0,484 & \\
\hline$\geq 19$ & $405(84,7)$ & 60 & 14,8 & 345 & 85,2 & & 1 \\
\hline$\leq 18$ & $73(15,3)$ & 13 & 17,8 & 60 & 82,2 & & $1,25(0,64-2,41)$ \\
\hline Situação conjugal & & & & & & 0,250 & \\
\hline Com parceiro fixo & $267(55,8)$ & 36 & 13,5 & 231 & 86,5 & & 1 \\
\hline Sem parceiro fixo & $211(44,2)$ & 37 & 17,5 & 174 & 82,5 & & $1,36(0,83-2,25)$ \\
\hline Escolaridade (anos de estudo) & & & & & & 0,410 & \\
\hline Ensino médio incompleto/completo/superior & $145(30,3)$ & 19 & 13,1 & 126 & 86,9 & & 1 \\
\hline Até ensino fundamental completo & $333(69,7)$ & 54 & 16,2 & 279 & 83,8 & & $1,28(0,73-2,25)$ \\
\hline Possui trabalho remunerado & & & & & & 0,060 & \\
\hline Sim & $128(26,8)$ & 13 & 10,2 & 115 & 89,8 & & 1 \\
\hline Não & $350(73,2)$ & 60 & 17,1 & 290 & 82,9 & & $1,83(0,97-3,46)$ \\
\hline Usuária de drogas ilícitas & & & & & & 0,001 & \\
\hline Não/ignorado & $376(78,7)$ & 47 & 12,5 & 329 & 87,5 & & 1 \\
\hline Sim & $102(21,3)$ & 26 & 25,5 & 76 & 74,5 & & $2,39(1,39-4,11)$ \\
\hline Frequentou pré-natal & & & & & & 0,004 & \\
\hline Sim & $396(82,8)$ & 52 & 13,1 & 344 & 86,9 & & 1 \\
\hline Não & $82(17,2)$ & 21 & 25,6 & 61 & 74,4 & & $2,28(1,28-4,05)$ \\
\hline Momento do diagnóstico de sífilis & & & & & & $<0,001$ & \\
\hline Pré-natal & $267(55,9)$ & 23 & 8,6 & 244 & 91,4 & & 1 \\
\hline Parto & $211(44,1)$ & 50 & 23,7 & 161 & 76,3 & & $3,29(1,93-5,61)$ \\
\hline Titulação do VDRL do parto & & & & & & 0,001 & \\
\hline$\leq 1: 8$ & $344(72,0)$ & 41 & 11,9 & 303 & 88,1 & & 1 \\
\hline$>1: 8$ & $134(28,0)$ & 32 & 23,9 & 102 & 76,1 & & $2,31(1,38-3,87)$ \\
\hline
\end{tabular}

Tabela 4. Assistência pré-natal de gestantes com sífilis associada ao desfecho de prematuridade (Fortaleza, Ceará, 2015).

\begin{tabular}{|c|c|c|c|c|c|c|c|}
\hline \multirow{3}{*}{ Variáveis } & \multirow{3}{*}{ n (\%) } & \multicolumn{4}{|c|}{ Prematuridade } & \multirow{3}{*}{$\mathbf{p}$} & \multirow{3}{*}{ OR bruto (IC95\%) } \\
\hline & & \multicolumn{2}{|c|}{ Sim } & \multicolumn{2}{|c|}{ Não } & & \\
\hline & & $\mathbf{n}$ & $\%$ & $\mathbf{n}$ & $\%$ & & \\
\hline Número de consultas & & & & & & 0,005 & \\
\hline$\geq 6$ & $209(52,8)$ & 18 & 8,6 & 191 & 91,4 & & 1 \\
\hline$<6$ & $187(47,2)$ & 34 & 18,2 & 153 & 81,8 & & $2,35(1,28-4,33)$ \\
\hline Início do pré-natal & & & & & & 0,092 & \\
\hline $1^{\circ}$ e $2^{\circ}$ trimestres & $354(89,4)$ & 43 & 12,1 & 311 & 87,9 & & 1 \\
\hline $3^{\circ}$ trimestre & $42(10,6)$ & 9 & 21,4 & 33 & 78,6 & & $1,97(0,88-4,40)$ \\
\hline Número de exames para sífilis no pré-natal & & & & & & 0,008 & \\
\hline Pelo menos um & $332(83,8)$ & 37 & 11,1 & 295 & 88,9 & & 1 \\
\hline Nenhum & $64(16,2)$ & 15 & 23,4 & 49 & 76,6 & & $2,44(1,24-4,77)$ \\
\hline Realizou dois VDRL no pré-natal & & & & & & 0,112 & \\
\hline Sim & $78(19,7)$ & 6 & 7,7 & 72 & 92,3 & & 1 \\
\hline Não/Ignorado & $318(80,3)$ & 46 & 14,5 & 272 & 85,5 & & $2,02(0,83-4,93)$ \\
\hline Tratamento da gestante no pré-natal & & & & & & $<0,001$ & \\
\hline Com penicilina benzatina & $180(45,4)$ & 11 & 6,1 & 169 & 93,9 & & 1 \\
\hline Outra droga/não tratada & $216(54,6)$ & 41 & 19,0 & 175 & 81,0 & & $3,59(1,79-7,23)$ \\
\hline
\end{tabular}


Tabela 5. Análise de regressão logística múltipla ajustada dos aspectos sociodemográficos, da atenção prénatal e parto de gestantes com sífilis associados ao desfecho de prematuridade (Fortaleza, Ceará, 2015).

\begin{tabular}{lccc}
\hline Variáveis & $\mathbf{n}(\%)$ & OR ajustado (IC95\%) & $\mathbf{p ~}$ \\
\hline Titulação do VDRL do parto & & 1 & 0,004 \\
$\quad \leq 1: 8$ & $344(72,0)$ & $2,46(1,33-4,53)$ & \\
$>1: 8$ & $134(28,0)$ & & $<0,001$ \\
Tratamento da gestante no pré-natal & & 1 \\
$\quad$ Com penicilina benzatina & $180(45,4)$ & $3,52(1,74-7,13)$ & \\
$\quad$ Outra droga/não tratada & $216(54,6)$ & & \\
\hline
\end{tabular}

2,28; IC95\%: 1,28-4,05), ter sido diagnosticada com sífilis no momento do parto ( $\mathrm{p}<0,001$; OR: 3,29; IC95\%: 1,93-5,61), ter apresentado titulação do VDRL do parto > 1:8 ( $p=0,001$; OR: 2,31; IC95\%: 1,38-3,87) (Tabela 3).

Ao analisar somente as gestantes que frequentaram o pré-natal, a prematuridade esteve associada a: ter recebido menos de seis consultas ( $p=0,005$; OR: 2,35; IC 95\%: 1,28-4,33), não ter realizado nenhum exame para sífilis ( $\mathrm{p}=0,008$; OR: 2,44; IC 95\%: 1,24-4,77), não ter sido tratada ou ter recebido tratamento com droga diferente da penicilina benzatina ( $<$ < 0,001; OR: 3,59; IC 95\%:1,79-7,23) (Tabela 4).

Na Tabela 5, é apresentada a análise de regressão logística múltipla, ajustada, dos aspectos sociodemográficos, da atenção pré-natal e partos de gestantes com sífilis associados ao desfecho de prematuridade. Encontrou-se que mulheres que apresentaram titulação do VDRL > 1:8 na ocasião do parto e aquelas que não foram tratadas ou receberam tratamento com droga diferente da penicilina apresentaram 2,46 (IC95\%: 1,33-4,53; p = 0,004) e 3,52 (IC95\%: 1,74-7,13; $\mathrm{p}<0,001$ ) vezes mais chance de ter bebês prematuros, respectivamente.

\section{DISCUSSÃO}

Neste estudo, encontrou-se desfecho de prematuridade em 15,3\% dos casos de SC, porcentagem superior às taxas estimadas para gestantes em geral descritas em estudo no Brasil: entre 7,7\% ${ }^{16}$ e $11,1 \%^{17}$. Gestantes que durante o pré-natal não foram tratadas ou receberam tratamento com drogas diferentes da penicilina benzatina, bem como as que apresentaram elevados títulos de VDRL no parto, tiveram mais desfecho de prematuridade.

Pode-se observar que uma quantidade significativa de gestantes com sífilis que tiveram bebês prematuros frequentou o pré-natal. Contudo, muitas oportunidades para prevenir a SC foram perdidas, situação evidenciada também em outro estudo, realizado em seis capitais brasileiras $^{18}$. O cuidado pré-natal pode impactar positivamente a saúde da gestante e evitar a mortalidade infantil ${ }^{19}$, e é um importante preditor para prevenir desfechos desfavoráveis relacionados à sífilis na gestação, desde que realizado com qualidade ${ }^{20,21}$.

Dentre a perda de oportunidades, destacam-se a falta de diagnóstico e falhas no tratamento das gestantes. Estudo de metanálise mostrou que as intervenções no pré-natal diminuem significativamente o risco de a gestante ter um resultado adverso devido à sífilis ${ }^{22}$. No entanto, no período deste estudo, o exame de rotina solicitado às gestantes era o VDRL, não havia coleta diária de sangue nas unidades, e o retorno dos resultados estava disponível somente 15 dias após a coleta, dificultando o acesso ao diagnóstico. Ademais, o teste rápido (TR) se encontrava em fase de implantação.

O TR para sífilis é uma estratégia que pode contribuir para aumentar a cobertura de testagem em gestantes, especialmente quando realizado na primeira consulta, possibilitando o tratamento imediato. Considerando que a grande maioria das gestantes recebeu duas ou mais consultas e iniciou o pré-natal antes do final do segundo trimestre de gestação, haveria tempo hábil para tratamento adequado caso tivessem realizado o TR. 
Ocorre que, durante o período de realização deste estudo, o Brasil vivenciou um problema grave de escassez de penicilina ${ }^{23}$, situação que contribuiu para que muitas gestantes não fossem tratadas ou recebessem medicamentos diferentes da penicilina benzatina. Estas gestantes apresentaram 3,52 vezes mais chances de terem bebês prematuros, situação também evidenciada em outros estudos realizados na China, que identificaram maiores chances de prematuridade e de outros desfechos em gestantes com sífilis não tratadas ou inadequadamente tratadas ${ }^{24,25}$.

A possibilidade de recorrência de escassez de penicilinas ${ }^{26}$ é preocupante, visto que a penicilina benzatina é a droga de escolha para tratamento da sífilis em gestantes por ser a única que atravessa a barreira transplacentária e trata o bebê ${ }^{23}$. Por esse motivo, é necessário desenvolver estudos que avaliem a eficácia de drogas alternativas ${ }^{27}$.

Nesse estudo, também foi identificado maior chance de prematuridade em gestantes cuja titulação do VDRL foi > 1:8 na ocasião do parto. Altos títulos de VDRL em gestantes durante o pré-natal e no momento do parto podem representar sífilis ativa, condição associada a desfechos graves de SC, dentre eles a prematuridade ${ }^{3,28}$. Por esse motivo, após o tratamento, o controle de cura da sífilis em gestantes deve ocorrer mensalmente, por meio do monitoramento da queda dos títulos do teste VDRL ${ }^{15}$.

Uma análise detalhada dos casos de gestantes que apresentaram altas titulações do VDRL na ocasião do parto mostrou que nenhuma mulher que recebeu tratamento completo (três doses de penicilina benzatina) teve bebê prematuro, reforçando a importância do diagnóstico e do tratamento de gestantes com sífilis em tempo hábil, prevenindo desfechos graves, como a prematuridade em decorrência da SC.

Este estudo tem como limitação o fato de ter analisado dados secundários, considerando que a falta de registro ou a baixa qualidade dos dados são comuns. Incluir a coleta em diferentes fontes de dados (fichas de notificação e prontuários) contribuiu sobremaneira para minimizar o problema. Ademais, a exclusão dos casos de natimortos, devido à falta de registro de variáveis importantes, e de gestantes que apresentavam agravos que pudessem interferir no desfecho de prematuridade por SC, podem ter subestimado o número de prematuros.

Os achados deste estudo evidenciam que a prematuridade decorrente da SC é evitável, desde que as gestantes com sífilis sejam tratadas adequadamente. Identificou-se também que fragilidades na assistência pré-natal estão associadas a este desfecho, o que ressalta a importância de implementar políticas públicas para melhorar a qualidade do pré-natal.

Nesse sentido, a atenção primária em saúde tem papel imprescindível para detectar a sífilis em gestantes e garantir a realização de exames e do tratamento. Esses esforços para prevenir a prematuridade por SC podem evitar repercussões importantes para o bebê, para a família e para o sistema de saúde.

\section{REFERÊNCIAS}

1. Domingues RMSM, Leal MC. Incidência de sífilis congênita e fatores associados à transmissão vertical da sífilis: dados do estudo Nascer no Brasil. Cad Saude Publica. 2016;32(6):e00082415. https://doi.org/10.1590/0102-311X00082415

2. Goldenberg RL, Culhane JF, lams JD, Romero R. Epidemiology and causes of preterm birth. Lancet. 2008;371(9606):75-84. https://doi.org/10.1016/S0140-6736(08)60074-4

3. Newman L, Kamb M, Hawkes S, Gomez G, Say L, Seuc A, et al. Global estimates of syphilis in pregnancy and associated adverse outcomes: analysis of multinational antenatal surveillance data. PLoS Med. 2013;10(2):e1001396. https://doi.org/10.1371/journal.pmed.1001396

4. Korenromp EL, Rowley J, Alonso M, Mello MB, Wijesooriya NS, Mahiané SG, et al. Global burden of maternal and congenital syphilis and associated adverse birth outcomes: estimates for 2016 and progress since 2012. PLOS One. 2019;14(2):e0211720. https://doi.org/10.1371/journal.pone.0211720 
5. Organización Panamericana de la Salud. Eliminación de la transmisión maternoinfantil del VIH y la sífilis en las Américas: actualización 2016. Washington, DC: OPS; 2017.

6. Ministério da Saúde (BR). Secretaria de Vigilância em Saúde. Boletim epidemiológico sífilis. Brasília, DF: Ministério da Saúde; 2019.

7. Secretaria de Saúde. Governo do Estado do Ceará. Boletim epidemiológico de sífilis. Fortaleza: Secretaria de Saúde; 2019.

8. Cardoso ARP, Araújo MAL, Cavalcante MS, Frota MA, De Melo SP. Análise dos casos de sífilis gestacional e congênita nos anos de 2008 a 2010 em Fortaleza, Ceará, Brasil. Cienc Saude Colet. 2018;23(2):563-74. https://doi.org/10.1590/1413-81232018232.01772016

9. Organización Mundial de la Salud. Orientaciones mundiales sobre los criterios y procesos para la validación de la eliminación de la transmisión maternoinfantil del VIH y la sifilis. Ginebra: OMS; 2015.

10. United Nations Organization. Transforming our world: the 2030 Agenda for Sustainable Development; 2015 [citado 2020 mai 13]. Disponível em: https://www.un.org/ga/search/view_ doc.asp?symbol=A/RES/70/1\&Lang=E.

11. França EB, Lansky S, Rego MAS, Malta DC, França JS, Renato Teixeira R, et al. Leading causes of child mortality in Brazil, in 1990 and 2015: estimates from the Global Burden of Disease study. Rev Bras Epidemiol. 2017;20 Suppl 1:46-60. https://doi.org/10.1590/1980-5497201700050005

12. Liu L, Oza S, Hogan D, Chu Y, Perin J, Zhu J, et al. Global, regional, and national causes of under-5 mortality in 2000-15: an updated systematic analysis with implications for the Sustainable Development Goals. Lancet. 2016;388(10063):3027-35. https://doi.org/10.1016/S0140-6736(16)31593-8

13. Katz J, Lee ACC, Kozuki N, Lawn JE, Cousens S, Blencowe H, et al. Mortality risk in preterm and small-for-gestational-age infants in low-income and middle-income countries: a pooled country analysis. Lancet. 2013;382(9890):417-25. https://doi.org/10.1016/S0140-6736(13)60993-9

14. Instituto Brasileiro de Geografia e Estatística - IBGE. Panorama Fortaleza. Rio de Janeiro: IBGE; c2017 [cited 2020 Dec 21]. Available from: https://cidades.ibge.gov.br/brasil/ce/ fortaleza/panorama.

15. Ministério da Saúde (BR). Protocolo clínico e diretrizes terapêuticas para atenção integral às pessoas com infecções sexualmente transmissíveis. Brasilia, DF: Ministério da Saúde; 2015.

16. Leal MDC, Esteves-Pereira AP, Viellas EF, Domingues RMSM, Gama SGND. Prenatal care in the Brazilian public health services. Rev Saude Publica. 2020;54:8. https://doi.org/10.11606/s1518-8787.2020054001458

17. Leal MC, Szwarcwald CL, Almeida PVB, Aquino EML, Barreto ML, Barros F, et al. Reproductive, maternal, neonatal and child health in the 30 years since the creation of the Unified Health System (SUS). Cienc Saude Colet. 2018;23(6):1915-28. https://doi.org/10.1590/1413-81232018236.03942018

18. Saraceni V, Pereira GFM, Silveira MF, Araújo MAL, Miranda AE. Vigilância epidemiológica da transmissão vertical da sífilis: dados de seis unidades federativas no Brasil. Rev Panam Salud Publica. Journal of Public Health. 2017;41:e44. https://doi.org/10.26633/RPSP.2017.44

19. Lima JC, Mingarelli AM, Segri NJ, Zavala AAZ, Takano AO. Estudo de base populacional sobre mortalidade infantil. Cienc Saude Colet. 2017;22(3):931-9. https://doi.org/10.1590/1413-81232017223.12742016

20. Plotzker RE, Murphy RD, Stoltey JE. Congenital syphilis prevention: strategies, evidence, and future directions. Sex Transm Dis. 2018;45 9S Suppl 1:29-37. https://doi.org/10.1097/OLQ.0000000000000846

21. Benzaken AS, Pereira GFM, Cunha ARCD, Souza FMA, Saraceni V. Adequacy of prenatal care, diagnosis and treatment of syphilis in pregnancy: a study with open data from Brazilian state capitals. Cad Saude Publica. 2019;36(1):e00057219. https://doi.org/10.1590/0102-311x00057219

22. Hawkes SJ, Gomez GB, Broutet N. Early antenatal care: does it make a difference to outcomes of pregnancy associated with syphilis? A systematic review and meta-analysis. PLoS One. 2013;8(2):e56713. https://doi.org/10.1371/journal.pone.0056713

23. Ministério da Saúde (BR). Nota Informativa Conjunta no 109/2015/GAB/SVS/MS, GAB/SCTIE/MS. Orienta a respeito da priorização da penicilina benzatina para sífilis em gestantes e penicilina cristalina para sífilis congênita no país e alternativas para o tratamento da sífilis. Brasília, DF: Ministério da Saúde; 2015. 
24. Zhang XH, Xu J, Chen D, Guo L, Qiu L. Effectiveness of treatment to improve pregnancy outcomes among women with syphilis in Zhejiang. Sex Transm Infect. 2016;92(7):537-41. https://doi.org/10.1136/sextrans-2015-052363

25. Qin JB, Feng TJ, Yang TB, Hong FC, Lan LN, Zhang CL, et al. Synthesized prevention and control of one decade for mother-to-child transmission of syphilis and determinants associated with congenital syphilis and adverse pregnancy outcomes in Shenzhen, South China. Eur J Clin Microbiol Infect Dis. 2014;33(12):2183-98. https://doi.org/10.1007/s10096-014-2186-8

26. Nurse-Findlay S, Taylor MM, Savage M, Mello MB, Saliyou M, Lavayen M, et al. Shortages of benzathine penicilina for prevention of mother-to-child transmission of syphilis: Na evaluation from multi-country surveys and stakeholder interviews. PLoS Med. 2017;14(12):e1002473. https://doi.org/10.1371/journal.pmed.1002473

27. Taylor MM, Kara EO, Araujo MAL, Silveira MS, Miranda AE, Coelho ICB, et al. Phase II trial evaluating the clinical efficacy of cefixime for treatment of active syphilis in non-pregnant women in Brazil (CeBra). BMC Infect Dis. 2020;20:405. https://doi.org/10.1186/s12879-020-04980-1

28. Wijesooriya NS, Rochat RW, Kamb ML, Turlapati P, Temmerman M, Broutet N, et al. Global burden of maternal and congenital syphilis in 2008 and 2012: a health systems modelling study. Lancet Glob Health. 2016;4(8):e525-33. https://doi.org/10.1016/S2214-109X(16)30135-8

Contribuição dos Autores: Concepção e planejamento do estudo: MALA, ABBE, AFBR. Coleta, análise e interpretação dos dados: MALA, ABBE, AFBR, GBSJ, AEM. Elaboração ou revisão do manuscrito: MALA, ABBE, AFBR, GBSJ, AEM. Aprovação da versão final: MALA, ABBE, AFBR, GBSJ, AEM. Responsabilidade pública pelo conteúdo do artigo: MALA, ABBE, AFBR, GBSJ, AEM.

Conflito de Interesses: Os autores declaram não haver conflito de interesses. 